\title{
Reduced Graphene Oxides as Carbocatalysts in Acceptorless Dehydrogenation of $\mathrm{N}$-Heterocycles
}

\author{
Andrés Mollar-Cuni, David Ventura-Espinosa, Santiago Martín, Hermenegildo García,* \\ and Jose A. Mata*
}

Cite This: ACS Catal. 2021, 11, 14688-14693

Read Online

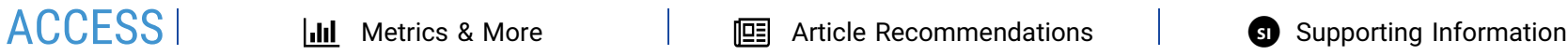

ABSTRACT: The catalytic properties of graphene-derived materials are evaluated in acceptorless dehydrogenation of $\mathrm{N}$-heterocycles. Among them, reduced graphene oxides (rGOs) are active (quantitative yields in $23 \mathrm{~h}$ ) under mild conditions $\left(130{ }^{\circ} \mathrm{C}\right.$ ) and act as efficient heterogeneous carbocatalysts. $\mathrm{rGO}$ exhibits reusability and stability at least during eight consecutive runs. Mechanistic investigations supported by experimental evidence (i.e., organic

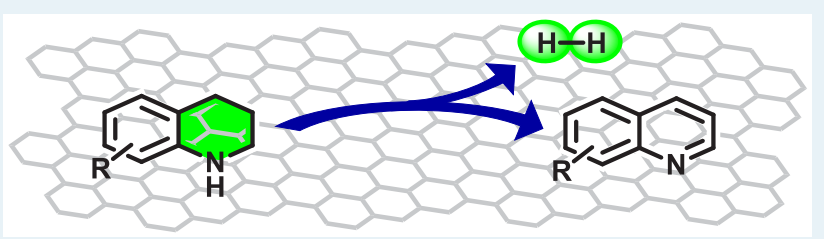
molecules as model compounds, purposely addition of metal impurities and selective functional group masking experiments) suggest a preferential contribution of ketone carbonyl groups as active sites for this transformation.

KEYWORDS: heterogeneous catalysis, carbocatalysis, reduced graphene oxide, N-heterocycles, dehydrogenation, graphene active sites

M etal-free catalysts might play an important role in the design of sustainable and environmentally friendly chemical transformations. ${ }^{1-4}$ The actual dependence of catalysis on metals, often precious and rare, is no longer sustainable, because of the cost, limited abundance, and depletion of metal sources. Development of metal-free catalysts based on abundant elements is a promising area of research. Organocatalysis is a well-established field in which well-defined active sites are present in organic molecules. ${ }^{5-7}$ Parallel to this, the use of heterogeneous carbonaceous materials is gaining interest, but the area is in its earlier development, in terms of understanding the reaction mechanisms, architecture of the active sites, and engineering of materials with single sites. ${ }^{8-10}$ In recent years, we have witnessed a rapid development of graphene-type materials as catalysts. ${ }^{11,12}$ Defective graphenes have shown catalytic activity in oxidation, ${ }^{13,14}$ reduction, ${ }^{15,16}$ and coupling reactions. ${ }^{17,18}$

In parallel, the hydrogenation and dehydrogenation of $\mathrm{N}$ heterocycles is an important reaction from the synthetic viewpoint. $N$-heterocycles are found in many highly addedvalue organic compounds. They are also considered as potential liquid organic hydrogen carriers (LOHCs) for hydrogen storage and release. ${ }^{19-21}$ One advantage of $\mathrm{N}$ heterocycles as LOHCs, compared to cycloalkanes, is the reduction of dehydrogenation enthalpy facilitating hydrogen release. $^{22-24}$ The success of $\mathrm{H}_{2}$ storage in organic compounds is dependent on the development of efficient, stable, and affordable catalysts. ${ }^{25-27}$ However, to the best of our knowledge, acceptorless dehydrogenation $(\mathrm{ADH})$ of tetrahydroquinolines using metal-free catalysts has not been reported. Previous examples describe the use of advanced nanostructures (e.g., nanodiamons) or activated mesoporous carbons in acceptorless gas-phase dehydrogenation of alkanes at high temperatures $\left(>500{ }^{\circ} \mathrm{C}\right) .{ }^{28-30}$ Alternatively, graphene oxide (GO) or activated carbon (AC) have been used in the oxidative dehydrogenation of $\mathrm{N}$-heterocycles with the concomitant formation of water or hydrogen peroxide (Figure 1). ${ }^{31-35}$ In this manuscript, we describe the activity of reduced graphene oxides (rGOs) as efficient and reusable carbocatalysts for the $\mathrm{ADH}$ of tetrahydroquinolines with the production of molecular hydrogen, a key reaction for on-board hydrogen release. Our research provides a general scope of the metal-free $\mathrm{ADH}$ carbocatalysts as well as evidence of the active sites responsible for this transformation.

The performance of $\mathrm{rGO}$ as carbocatalyst in $\mathrm{ADH}$ of $\mathrm{N}$ heterocycles was first evaluated using 1,2,3,4-tetrahydroquinoline (THQ $1 \mathbf{1 H}$ ) as a model substrate under oxygen-free conditions (see Table 1, as well as Figure S1 in the Supporting Information (SI)). Our first concern was to determine whether rGO was acting as a true carbocatalyst or as a stoichiometric reagent. ${ }^{36}$ First of all, we confirmed that dehydrogenation of THQ did not occur without rGO (Table 1, entry 1 ). In the presence of $\mathrm{rGO}$, quinoline is obtained under different solvents and reaction conditions, indicating that the process is general. $\mathrm{ADH}$ of $1 \mathrm{H}$ afforded quinoline (1D) with the concomitant release of two molecules of $\mathrm{H}_{2}$. Hydrogen was qualitatively analyzed using a microGC. The $\mathrm{ADH}$ of $\mathrm{N}$-heterocycles is an

Received: October 8, 2021

Revised: November 17, 2021

Published: November 23, 2021 


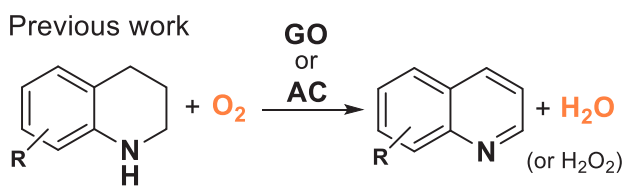

Oxidative Dehydrogenation (ODH)

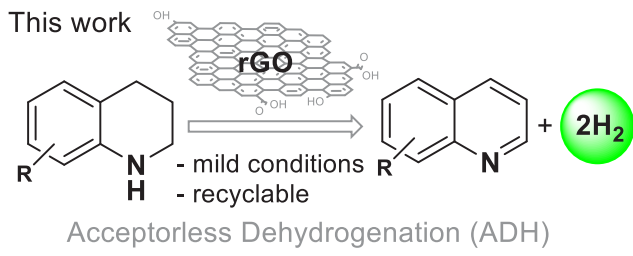

Figure 1. Differences between oxidative and acceptorless dehydrogenation of $\mathrm{N}$-heterocycles. The latter is important for $\mathrm{H}_{2}$ storage. In $\mathrm{ADH}, \mathrm{H}_{2}$ gas is released while in $\mathrm{ODH}$ hydrogen is transferred to oxygen and released as $\mathrm{H}_{2} \mathrm{O}$. [Legend: $\mathrm{GO}$, graphene oxide; $A C$, activated carbon, and rGO, reduced graphene oxide.]

Table 1. Catalytic Activity of rGO in ADH of 1,2,3,4Tetrahydroquinoline (1H) under Various Reaction Conditions $^{a}$
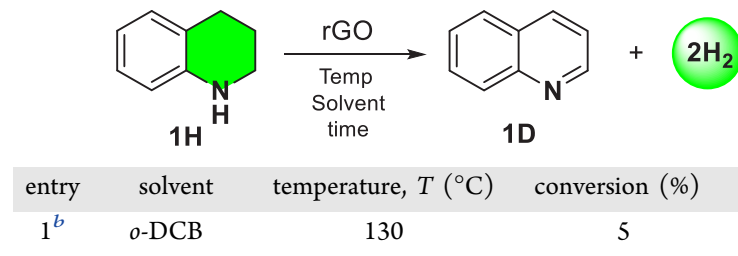

$\begin{array}{clccc}\text { entry } & \text { solvent } & \text { temperature, } T\left({ }^{\circ} \mathrm{C}\right) & \text { conversion }(\%) & \text { yield (\%) } \\ 1^{b} & o \text {-DCB } & 130 & 5 & 1 \\ 2 & o \text {-DCB } & 130 & 91 & 85 \\ 3 & o \text {-DCB } & 110 & 78 & 67 \\ 4 & \text { DMF } & 130 & 74 & 58 \\ 5 & \text { toluene } & 110 & 14 & 9 \\ 6 & n \text {-decane } & 130 & 81 & 64 \\ 7 & \text { DIPB } & 130 & 79 & 58 \\ 8^{d} & o \text {-DCB } & 130 & 10 & \text { n.d. }\end{array}$

${ }^{a}$ Reaction conditions: 1,2,3,4-tetrahydroquinoline ( $\left.0.15 \mathrm{mmol}\right), \mathrm{rGO}$ $(15 \mathrm{mg})$, solvent $(1 \mathrm{~mL})$ for $23 \mathrm{~h}$. Evolution of starting material (conversion) obtained by GC/FID using 1,3,5-trimethoxybenzene as internal standard and product formation (yield) obtained by ${ }^{1} \mathrm{H}$ NMR analysis. ${ }^{b}$ Without rGO. ${ }^{c}$ Legend: o-DCB: ortho-dichlorobenzene; DMF: $N, N$-dimethylformamide: and DIPB, 1,3-diisopropylbenzene. ${ }^{d}$ Closed system.

endergonic reaction, in contrast to the $\mathrm{ODH}$. The driving force of $\mathrm{ADH}$ is the removal of hydrogen from the reaction media. In fact, when the dehydrogenation reaction is performed in a closed system, no product formation occurred (Table 1, entry $8)$. Then, the influence of catalyst loading in the dehydrogenation of 8-methoxytetrahydroquinoline $(7 \mathbf{H})$ was assessed (Figure S1). The apparent reaction rates are dependent on the amount of $\mathrm{rGO}$, and the reaction profiles suggest a catalytic nature for the reaction. For instance, lowering the catalyst loading to $5 \mathrm{mg}$ considerably decreased the apparent reaction rate, but still a good yield (68\%) could be obtained after $23 \mathrm{~h}$. The selectivity and the presence of other reaction products was evaluated through analogous experiments using deuterated $o$ DCB and by analyzing the reaction mixture without any purification process (Figures S2-S4 in the SI). By monitoring the reaction progress by ${ }^{1} \mathrm{H}$ NMR spectroscopy, it could be seen how the starting material $(\mathbf{3 H})$ is converted to the corresponding dehydrogenated product (3D) without the formation of other products. This confirmed the high selectivity of $\mathrm{ADH}$ of THQs toward quinolines using $\mathrm{rGO}$ as a carbocatalyst.

Next, we investigated the scope and limitations of $\mathrm{N}$ heterocycle dehydrogenation, using $\mathrm{rGO}$ as a carbocatalyst (see Table 2). The reactions were monitored by GC, and the

Table 2. Scope of ADH of $\mathrm{N}$-Heterocycles ${ }^{a}$

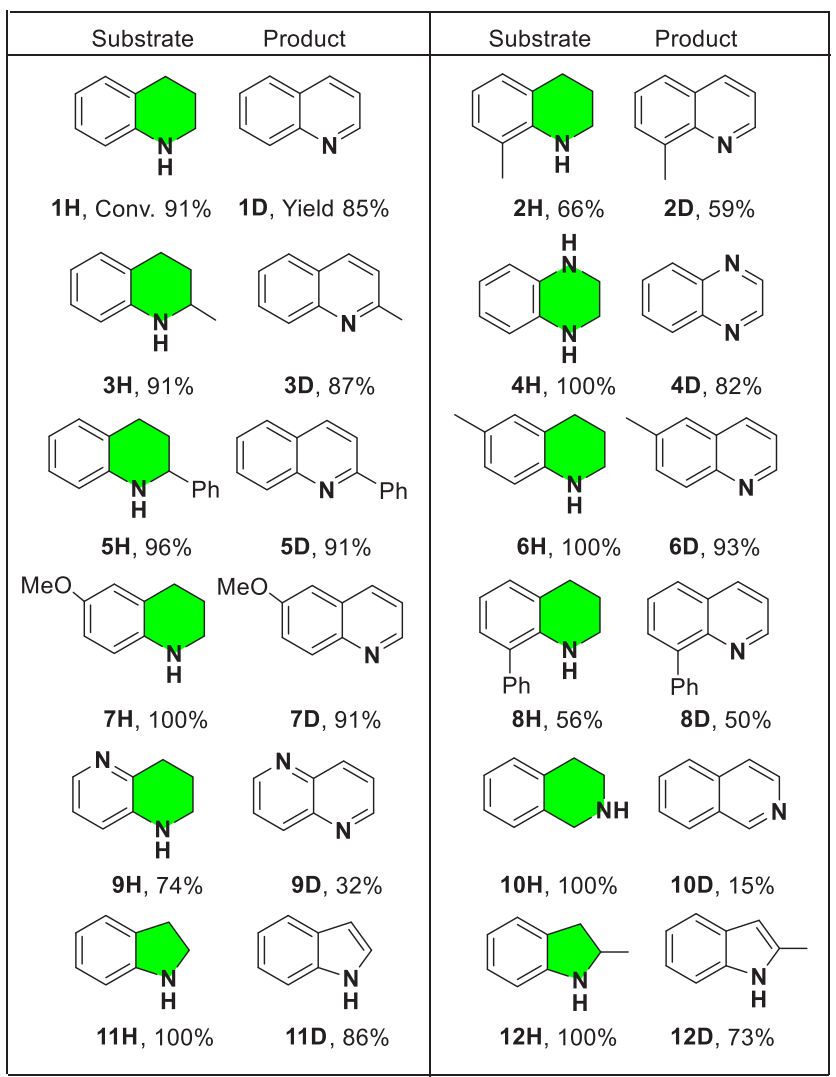

${ }^{a}$ Reaction conditions: Substrate $(0.15 \mathrm{mmol}), 15 \mathrm{mg}$ of $\mathrm{rGO}, o-\mathrm{DCB}$ $(1 \mathrm{~mL})$ at $130{ }^{\circ} \mathrm{C}$ for $23 \mathrm{~h}$. The number under the starting material corresponds to conversion obtained by GC/FID and the number under the product corresponds to the isolated product yield after purification. See the SI for the reaction profiles and details. " $\mathrm{H}$ " denotes hydrogenated; " $D$ " denotes "dehydrogenated".

activity was compared using apparent reaction rates. (See the SI for details.) rGO is an efficient carbocatalyst for a variety of substituted THQs and indolines. Methyl $(\mathbf{3 H})$ or phenyl $(\mathbf{5 H})$ substitution at the 2-position of THQ showed similar rates and afforded quantitative yields after $23 \mathrm{~h}$. Introduction of different groups at the 6-position $(6 \mathrm{H}$ and $7 \mathrm{H})$ does not influence the formation of quinolines, and similar reaction rates were obtained. A substantial limitation in ADH of THQ substituted at the 8-position was observed. The presence of a methyl $(2 \mathrm{H})$ or a phenyl $(8 \mathrm{H})$ reduced the yield of $2 \mathrm{D}$ and $8 \mathrm{D}$ to $56 \%$ and $50 \%$, respectively, probably because of steric effects. The same procedure was used for dehydrogenation of tetrahydroisoquinoline $(\mathbf{1 0 H})$. Under these conditions, full conversion of $10 \mathrm{H}$ was also obtained, but only $15 \%$ yield corresponds to isoquinoline (10D): the rest $(\sim 70 \%$ yield $)$ is the monodehydrogenated product. Monitoring of the temporal reaction profile indicates that double dehydrogenation of $10 \mathrm{H}$ requires longer reaction times (Figure S12 in the SI). A fast reaction and quantitative conversion was obtained for tetrahydroquinoxaline $(4 \mathrm{H})$ containing two nitrogen groups (92\% 
conversion over $8 \mathrm{~h}$ ). This result is in agreement with previous theoretical calculations, revealing that the dehydrogenation is thermodynamically favored by increasing the number of $\mathrm{N}$ atoms in the $\mathrm{N}$-heterocycles. ${ }^{23}$ An important limitation was found for tetrahydronaphthyridine $(\mathbf{9 H})$, while a good conversion of $76 \%$ was observed; in contrast, the product 9D could only be obtained in a low yield (32\%), because of product degradation. Further studies with five-membered ring $\mathrm{N}$-heterocycles (indolines) showed faster reactions. For instance, indoline $(11 \mathrm{H})$ and 2 -methylindoline $(12 \mathrm{H})$ were fully dehydrogenated to the corresponding indole (11D) or 2methylindole (12D) within $<10 \mathrm{~h}$.

The performance of rGO as a carbocatalyst was further evaluated by recycling experiments. The temporal reaction profile provides valuable information of activity and stability. The rGO was removed from the solution after each run by decantation, washed with $\mathrm{MeOH}$, and reused without any preactivation process. The data presented in Figure 2 are the

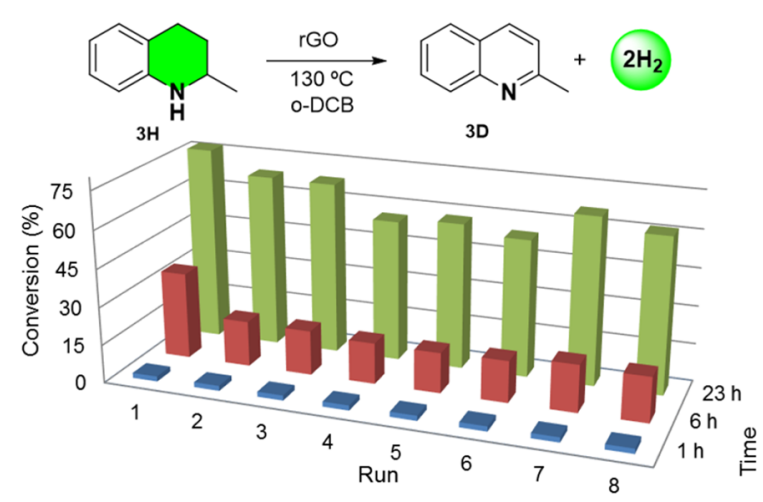

Figure 2. Activity of rGO as carbocatalyst for $\mathrm{ADH}$ of $\mathrm{N}$-heterocycles. Reaction conditions: Tetrahydroquinaldine $(3 \mathrm{H}, 0.3 \mathrm{mmol})$, catalyst loading $(30 \mathrm{mg}$ of $\mathrm{rGO})$, with $o$-DCB $(2.0 \mathrm{~mL})$ as solvent at $130^{\circ} \mathrm{C}$. Conversion was determined by gas chromatography/flame ionization detection (GC/FID) and using 1,3,5-trimethoxybenzene as an internal standard.

average of two independent reactions. These results show some decrease in activity from run 1 to run 2 and then, from run 4 to run 6 . However, the activity is recovered from runs 7 and 8 . These fluctuations are due to the imperfect experimental working procedure with $\mathrm{rGO}$, rather than a real tendency to deactivation. In any case, the catalytic activity of rGO is maintained for eight consecutive runs. Note that we have not observed any sign of catalyst deactivation. This fact suggests that $\mathrm{rGO}$ is a stable carbocatalyst. After the recycling experiments, the spent rGO was analyzed by high-resolution transmission electron microscopy (HRTEM), X-ray photoelectron spectroscopy (XPS), elemental analysis, and Raman spectroscopy to determine any changes in the morphology and composition (Figures S15-S17 in the SI). The only subtle difference is the presence of more wrinkles in the spent rGO carbocatalyst. We believe that the presence of these wrinkles is not directly related to the catalytic activity, but rather a consequence of the use of rGO in multiple experiments. XPS spectra of the $\mathrm{C} 1 \mathrm{~s}$ and $\mathrm{O}$ 1s bands for the fresh and spent rGO show a similar peak shapes and at the same binding energies (see Figure S16). Deconvolution provides similar contributions of different bonding modes $(\mathrm{C}-\mathrm{C}, \mathrm{C}-\mathrm{N}, \mathrm{C}-\mathrm{O}, \mathrm{C}=\mathrm{O}$, and $\mathrm{HO}-\mathrm{C}=\mathrm{O}$ ) for the fresh and spent rGO. The oxygen/ carbon ratio for the fresh rGO sample was 0.12 and for the spent rGO (8 runs) was 0.13 . These results confirm that, during the catalytic reaction, there is no increase or decrease in oxygen content. Elemental composition by combustion analyses confirmed that the oxygen/carbon ratio is maintained after exhaustive recycling (Table S3 in the SI). The Raman spectra provided the characteristic graphene pattern with $D, G$, and $2 \mathrm{D}$ bands. The relative intensity $\left(I_{\mathrm{D}} / I_{\mathrm{G}}\right)$ of $\mathrm{D}$ and $\mathrm{G}$ bands is preserved after eight runs, indicating the high stability of rGO (see Figure S17). The available characterization data before and after the recycling experiment indicates that $\mathrm{rGO}$ is not altered during the dehydrogenation of $\mathrm{N}$-heterocycles.

Mechanistic studies of catalytic reactions using carbonaceous materials is a difficult task, because of the ill-defined nature of the active sites. ${ }^{37}$ To get information about the catalytic active sites of $\mathrm{rGO}$, experiments were performed to address the influence of residual metals, the use of organic molecules as models of the active centers, and the selective masking of functional groups. We first assessed the catalytic activity of different graphene-related materials including rGO from different commercial suppliers. The results (Figure S25) showed that graphene $(G)$ and graphite are not active in the dehydrogenation of $\mathrm{N}$-heterocycles. GO is highly active at low conversions, but then it deactivates fast and, after a prolonged reaction time, only $63 \%$ yield is obtained. In contrast, all rGO materials used show a remarkable activity independently if they are obtained via thermal or chemical reduction. This observation indicates that the presence of vacancies or holes, which are more common in thermal rGO, or the presence of adventitious sites, such as $\mathrm{N}$ atoms in chemical reduction by hydrazine, are not necessary for the catalytic activity. The results derived from different carbonaceous materials indicate that the functional groups and the $\mathrm{sp}^{2}$ character of the basal plane of carbonaceous materials play a role as active sites in the catalytic reaction.

Then, we analyzed the potential catalytic activity of metal impurities in the carbonaceous materials. Carbonaceous materials are predominantly obtained from graphite that contains several metal impurities present at trace levels. ${ }^{38-40}$ These impurities could have an impact in the catalytic properties of graphene. In addition, the preparation of GO and $\mathrm{rGO}$ involves the use of oxidant/reducing agents, some of which can contain metals that may remain in the final samples, even at low concentrations. Common metal impurities found in rGO are iron and manganese. We evaluated the catalytic activity in dehydrogenation of $N$-heterocycles by adding known amounts of these metals in minute concentrations. The reaction progress in three parallel reactions-containing no added metal, with $0.5 \mathrm{mg}$ of $\mathrm{M}^{2+}$, and with $1 \mathrm{mg}$ of $\mathrm{M}^{2+}-$ were monitored (see Figures S27 and S28 in the SI). The results show that the three curves overlapped for $\mathrm{Mn}^{2+}$ or $\mathrm{Fe}^{2+}$, suggesting that the catalytic effect induced by the presence of these metal ions at these concentrations is negligible.

We also used a series of organic molecules as models of the active sites to assess the role of different functional groups $(-\mathrm{OH},-\mathrm{COOH}$, and $\mathrm{C}=\mathrm{O})$ in the $\mathrm{ADH}$ of $\mathrm{N}$-heterocycles (see Figure S29 in the SI). The use of simple organic molecules for mimicking the role oxygenated groups at the surface of carbon materials is gaining interest, because of the relevant information provided. ${ }^{41,42}$ The results show that model molecules containing carboxylic acids and carbonyl groups somehow promote the dehydrogenation of $\mathrm{N}$-heterocycles (see Table S5 in the SI). For instance, the yield of 2methyltetraquinoline (3D) is $19 \%$ and $34 \%$ when using 
benzoic acid (A) and pyrene-4,5-dione (H), respectively. Other compounds, such as benzoquinone and phenol, also exhibit some activity. The results also suggested that the chemical environment of the functional groups is important, as we observed differences between functional groups attached to benzene or pyrene scaffolds. The activity of the model molecules suggests that carboxylic acids, hydroxy groups, and carbonyl groups present on rGO are potential catalytic active sites. We then used modified rGO samples in which certain functional groups have been selectively masked to provide further information on active sites. Carboxylic acids, ketone groups, and phenol groups of rGO were selectively masked using established procedures (see Scheme S1 in the SI). ${ }^{43,44}$ The catalytic activity of the parent $\mathrm{rGO}$ and the masked materials $\mathrm{rGO}^{\mathrm{COOH}}$, $\mathrm{rGO}^{\mathrm{CO}}$, and $\mathrm{rGO}^{\mathrm{OH}}$ (where the superscript indicates the masked group) was evaluated in dehydrogenation of tetrahydroquinaldine $(3 \mathbf{H})$. The results reveal that samples without carboxylic groups or phenolic moieties exhibit lower apparent reaction rates than unprotected rGO. A more remarkable decrease in the dehydrogenation rates occurs upon masking the carbonyl groups (see Figure S32 in the SI). The results of masking experiments suggest that the carbonyl groups are the prevalent active sites that, together with the lesser activity of carboxylic and hydroxyl groups, are responsible for the performance of rGO.

Previous studies on the use of $\mathrm{rGO}$ as carbocatalyst have already claimed the role of carbonyl groups (quinone type) as active sites. $^{41,45,46}$ Based on present experimental evidence, a plausible mechanism for the $\mathrm{ADH}$ of $\mathrm{N}$-heterocycles is proposed in which the role of carbonyl groups is emphasized as previously observed in $\mathrm{ODH}$ (Figure 3). ${ }^{47-49}$ According to

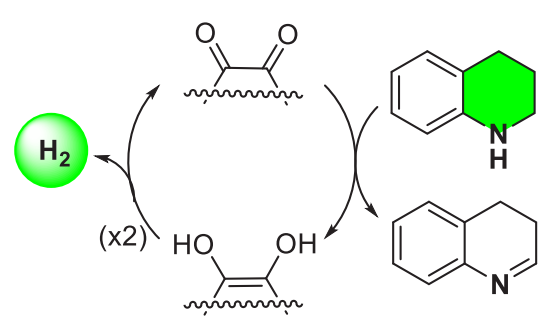

Figure 3. Plausible mechanism in the dehydrogenation of $N$ heterocycles, showing the role of carbonyl groups.

this proposal, dehydrogenation of the $N$-heterocycles would occur with the concomitant reduction of carbonyl groups that, in a subsequent step, would release molecular hydrogen.

Graphene oxide (GO) is an efficient metal-free carbocatalyst for oxidative dehydrogenation (OHD) reactions. In this manuscript, reduced graphene oxide (rGO) behaves as a suitable carbocatalyst to promote, without metal assistance, a more challenging thermodynamically uphill reaction, namely, the acceptorless dehydrogenation $(\mathrm{ADH})$ of $N$-heterocycles. In order the reaction to proceed, $\mathrm{H}_{2}$ must be removed from the system, while the catalyst must be very efficient in establishing equilibrium concentrations. rGOs of various origins are equally active as catalysts promoting dehydrogenation of a wide range of $\mathrm{N}$-heterocycles. Available catalytic data indicate that possible metal impurities are not involved in the process and that the most likely active sites are quinone-like carbonylic groups. In this way, the present results represent a step forward toward the sustainability for on-board hydrogen release that could be applicable in massive scale without mining or depletion of limited metal resources.

\section{ASSOCIATED CONTENT}

\section{Supporting Information}

The Supporting Information is available free of charge at https://pubs.acs.org/doi/10.1021/acscatal.1c04649.

Experimental details, synthetic procedures, mechanistic protocols, and full characterization of the carbonaceous materials (PDF)

\section{AUTHOR INFORMATION}

\section{Corresponding Authors}

Jose A. Mata - Institute of Advanced Materials (INAM), Centro de Innovación en Química Avanzada (ORFEO-CINQA), Universitat Jaume I, 12006 Castellón, Spain; 이이.org/0000-0001-9310-2783; Email: jmata@ uji.es

Hermenegildo García - Instituto de Tecnología Química, Consejo Superior de Investigaciones Cientificas-Universitat Politènica de Valencia, 46022 Valencia, Spain; ○ orcid.org/0000-0002-9664-493X; Email: hgarcia@ qim.upv.es

\section{Authors}

Andrés Mollar-Cuni - Institute of Advanced Materials (INAM), Centro de Innovación en Química Avanzada (ORFEO-CINQA), Universitat Jaume I, 12006 Castellón, Spain

David Ventura-Espinosa - Institute of Advanced Materials (INAM), Centro de Innovación en Química Avanzada (ORFEO-CINQA), Universitat Jaume I, 12006 Castellón, Spain

Santiago Martín - Instituto de Nanociencia y Materiales de Aragón (INMA), CSIC-Universidad de Zaragoza, Zaragoza 50009, Spain; Departamento de Química Física, Universidad de Zaragoza, 50009 Zaragoza, Spain; Laboratorio de Microscopias Avanzadas (LMA), Universidad de Zaragoza, 50018 Zaragoza, Spain; 이잉.org/0000-0001-91933874

Complete contact information is available at:

https://pubs.acs.org/10.1021/acscatal.1c04649

\section{Author Contributions}

The manuscript was written through contributions of all authors. All authors have given approval to the final version of the manuscript.

\section{Funding}

Supported by MCIN/AEI/10.13039/501100011033/FEDER (Grant Nos. RTI2018-098237-B-C21, RTI2018-098237-BC22, and PID2019-105881RB-I00), Generalitat Valenciana (No. PROMETEU/2020/028), and Universitat Jaume I (No. UJI-B2018-23).

\section{Notes}

The authors declare no competing financial interest.

\section{ACKNOWLEDGMENTS}

Thanks to Grant Nos. RTI2018-098237-B-C21, RTI2018098237-B-C22, and PID2019-105881RB-I00, financed by MCIN/AEI/10.13039/501100011033/FEDER, "Una manera de hacer Europa”, Generalitat Valenciana (No. PROMETEU/ 2020/028) and Universitat Jaume I (No. UJI-B2018-23). S.M. 
acknowledges DGA/fondos FEDER (construyendo Europa desde Aragón) for funding the research group Platón (E31_20R). A.M.-C. thanks Generalitat Valenciana for Grant No. ACIF/2019/100). The authors thank "Servei Central d"Instrumentació Científica (SCIC) de la Universitat Jaume I' and Dr. G. Antorrena for technical support in XPS studies.

\section{ABBREVIATIONS}

rGO = reduced graphene oxide

$\mathrm{GO}=$ graphene oxide

$\mathrm{ADH}=$ acceptorless dehydrogenation

$\mathrm{ODH}=$ oxidative dehydrogenation

\section{REFERENCES}

(1) Dalko, P. I.; Moisan, L. In the Golden Age of Organocatalysis. Angew. Chem., Int. Ed. 2004, 43 (39), 5138-5175.

(2) MacMillan, D. W. C. The Advent and Development of Organocatalysis. Nature 2008, 455 (7211), 304-308.

(3) Su, D. S.; Wen, G.; Wu, S.; Peng, F.; Schlögl, R. Carbocatalysis in Liquid-Phase Reactions. Angew. Chem., Int. Ed. 2017, 56 (4), 936964.

(4) Figueiredo, J. L.; Pereira, M. F. R. The Role of Surface Chemistry in Catalysis with Carbons. Catal. Today 2010, 150 (1-2), 2-7.

(5) Chua, C. K.; Pumera, M. Carbocatalysis: The State of "MetalFree” Catalysis. Chem. - Eur. J. 2015, 21 (36), 12550-12562.

(6) Dreyer, D. R.; Bielawski, C. W. Carbocatalysis: Heterogeneous Carbons Finding Utility in Synthetic Chemistry. Chem. Sci. 2011, 2 (7), 1233-1240.

(7) Titirici, M.-M.; White, R. J.; Brun, N.; Budarin, V. L.; Su, D. S.; del Monte, F.; Clark, J. H.; MacLachlan, M. J. Sustainable Carbon Materials. Chem. Soc. Rev. 2015, 44 (1), 250-290.

(8) Espinosa, J. C.; Navalón, S.; Primo, A.; Moral, M.; Sanz, J. F.; Álvaro, M.; García, H. Graphenes as Efficient Metal-Free Fenton Catalysts. Chem. - Eur. J. 2015, 21 (34), 11966-11971.

(9) Navalon, S.; Dhakshinamoorthy, A.; Alvaro, M.; Antonietti, M.; García, H. Active Sites on Graphene-Based Materials as Metal-Free Catalysts. Chem. Soc. Rev. 2017, 46 (15), 4501-4529.

(10) Lombardi, L.; Bandini, M. Graphene Oxide as a Mediator in Organic Synthesis: A Mechanistic Focus. Angew. Chem., Int. Ed. 2020, 59 (47), 20767-20778.

(11) Navalon, S.; Dhakshinamoorthy, A.; Alvaro, M.; Garcia, H. Carbocatalysis by Graphene-Based Materials. Chem. Rev. 2014, 114 (12), 6179-6212.

(12) Su, C.; Loh, K. P. Carbocatalysts: Graphene Oxide and Its Derivatives. Acc. Chem. Res. 2013, 46 (10), 2275-2285.

(13) Axet, M. R.; Dechy-Cabaret, O.; Durand, J.; Gouygou, M.; Serp, P. Coordination Chemistry on Carbon Surfaces. Coord. Chem. Rev. 2016, 308, 236-345.

(14) Dreyer, D. R.; Jia, H.-P.; Bielawski, C. W. Graphene Oxide: A Convenient Carbocatalyst for Facilitating Oxidation and Hydration Reactions. Angew. Chem., Int. Ed. 2010, 49 (38), 6813-6816.

(15) Espinosa, J. C.; Navalón, S.; Álvaro, M.; García, H. Reduced Graphene Oxide as a Metal-Free Catalyst for the Light-Assisted Fenton-Like Reaction. Chem CatChem 2016, 8 (16), 2642-2649.

(16) Machado, B. F.; Serp, P. Graphene-Based Materials for Catalysis. Catal. Sci. Technol. 2012, 2 (1), 54-75.

(17) Gao, Y.; Ma, D.; Wang, C.; Guan, J.; Bao, X. Reduced Graphene Oxide as a Catalyst for Hydrogenation of Nitrobenzene at Room Temperature. Chem. Commun. 2011, 47 (8), 2432-2434.

(18) Hu, H.; Xin, J. H.; Hu, H.; Wang, X. Structural and Mechanistic Understanding of an Active and Durable Graphene Carbocatalyst for Reduction of 4-Nitrophenol at Room Temperature. Nano Res. 2015, 8 (12), 3992-4006.

(19) Acocella, M. R.; Mauro, M.; Falivene, L.; Cavallo, L.; Guerra, G. Inverting the Diastereoselectivity of the Mukaiyama-Michael
Addition with Graphite-Based Catalysts. ACS Catal. 2014, 4 (2), 492-496.

(20) Su, C.; Acik, M.; Takai, K.; Lu, J.; Hao, S. J.; Zheng, Y.; Wu, P.; Bao, Q.; Enoki, T.; Chabal, Y. J.; Loh, K. P. Probing the Catalytic Activity of Porous Graphene Oxide and the Origin of This Behaviour. Nat. Commun. 2012, 3, 1-9.

(21) Preuster, P.; Papp, C.; Wasserscheid, P. Liquid Organic Hydrogen Carriers (LOHCs): Toward a Hydrogen-Free Hydrogen Economy. Acc. Chem. Res. 2017, 50 (1), 74-85.

(22) Crabtree, R. H. Hydrogen Storage in Liquid Organic Heterocycles. Energy Environ. Sci. 2008, 1 (1), 134-138.

(23) Zheng, J.; Zhou, H.; Wang, C.-G.; Ye, E.; Xu, J. W.; Loh, X. J.; $\mathrm{Li}$, Z. Current Research Progress and Perspectives on Liquid Hydrogen Rich Molecules in Sustainable Hydrogen Storage. Energy Storage Mater. 2021, 35, 695-722.

(24) Crabtree, R. H. Nitrogen-Containing Liquid Organic Hydrogen Carriers: Progress and Prospects. ACS Sustainable Chem. Eng. 2017, 5 (6), 4491-4498.

(25) Clot, E.; Eisenstein, O.; Crabtree, R. H. Computational Structure-Activity Relationships in $\mathrm{H}_{2}$ Storage: How Placement of $\mathrm{N}$ Atoms Affects Release Temperatures in Organic Liquid Storage Materials. Chem. Commun. 2007, 22 (22), 2231-2233.

(26) Zhang, Y.; Wang, J.; Zhou, F.; Liu, J. An Effective Strategy for Hydrogen Supply: Catalytic Acceptorless Dehydrogenation of $\mathrm{N}$ Heterocycles. Catal. Sci. Technol. 2021, 11 (12), 3990-4007.

(27) Rao, P. C.; Yoon, M. Potential Liquid-Organic Hydrogen Carrier (LOHC) Systems: A Review on Recent Progress. Energies 2020, 13 (22), 6040-6063.

(28) Sinigaglia, T.; Lewiski, F.; Santos Martins, M. E.; Mairesse Siluk, J. C. Production, Storage, Fuel Stations of Hydrogen and Its Utilization in Automotive Applications-a Review. Int. J. Hydrogen Energy 2017, 42 (39), 24597-24611.

(29) Teichmann, D.; Arlt, W.; Wasserscheid, P. Liquid Organic Hydrogen Carriers as an Efficient Vector for the Transport and Storage of Renewable Energy. Int. J. Hydrogen Energy 2012, 37 (23), 18118-18132.

(30) Zhang, J.; Su, D. S.; Blume, R.; Schlögl, R.; Wang, R.; Yang, X.; Gajović, A. Surface Chemistry and Catalytic Reactivity of a Nanodiamond in the Steam-Free Dehydrogenation of Ethylbenzene. Angew. Chem., Int. Ed. 2010, 49 (46), 8640-8644.

(31) Liu, L.; Deng, Q.-F.; Liu, Y.-P.; Ren, T.-Z.; Yuan, Z.-Y. $\mathrm{HNO}_{3}$ Activated Mesoporous Carbon Catalyst for Direct Dehydrogenation of Propane to Propylene. Catal. Commun. 2011, 16 (1), 81-85.

(32) Liu, L.; Deng, Q.-F.; Agula, B.; Ren, T.-Z.; Liu, Y.-P.; Zhaorigetu, B.; Yuan, Z.-Y. Synthesis of Ordered Mesoporous Carbon Materials and Their Catalytic Performance in Dehydrogenation of Propane to Propylene. Catal. Today 2012, 186 (1), 35-41.

(33) Zhang, J.; Chen, S.; Chen, F.; Xu, W.; Deng, G.-J.; Gong, H. Dehydrogenation of Nitrogen Heterocycles Using Graphene Oxide as a Versatile Metal-Free Catalyst under Air. Adv. Synth. Catal. 2017, 359 (14), 2358-2363.

(34) Hayashi, M. Oxidation Using Activated Carbon and Molecular Oxygen System. Chem. Rec. 2008, 8 (4), 252-267.

(35) Kawashita, Y.; Hayashi, M. Synthesis of Heteroaromatic Compounds by Oxidative Aromatization Using an Activated Carbon/Molecular Oxygen System. Molecules 2009, 14 (8), 30733093.

(36) Enders, L.; Casadio, D. S.; Aikonen, S.; Lenarda, A.; Wirtanen, T.; Hu, T.; Hietala, S.; Ribeiro, L. S.; Pereira, M. F. R.; Helaja, J. Air Oxidized Activated Carbon Catalyst for Aerobic Oxidative Aromatizations of N-Heterocycles. Catal. Sci. Technol. 2021, 11 (17), 59625972.

(37) Pereira, M. F. R.; Órfão, J. J. M.; Figueiredo, J. L. Oxidative Dehydrogenation of Ethylbenzene on Activated Carbon Catalysts. I. Influence of Surface Chemical Groups. Appl. Catal., A 1999, 184 (1), $153-160$.

(38) Presolski, S.; Pumera, M. Graphene Oxide: Carbocatalyst or Reagent? Angew. Chem., Int. Ed. 2018, 57 (51), 16713-16715. 
(39) Navalón, S.; Herance, J. R.; Álvaro, M.; García, H. General Aspects in the Use of Graphenes in Catalysis. Mater. Mater. Horiz. 2018, 5 (3), 363-378.

(40) Ambrosi, A.; Chee, S. Y.; Khezri, B.; Webster, R. D.; Sofer, Z.; Pumera, M. Metallic Impurities in Graphenes Prepared from Graphite Can Dramatically Influence Their Properties. Angew. Chem., Int. Ed. 2012, 51 (2), 500-503.

(41) Wang, L.; Ambrosi, A.; Pumera, M. Metal-Free" Catalytic Oxygen Reduction Reaction on Heteroatom-Doped Graphene Is Caused by Trace Metal Impurities. Angew. Chem., Int. Ed. 2013, 52 (51), 13818-13821.

(42) Ambrosi, A.; Chua, C. K.; Khezri, B.; Sofer, Z.; Webster, R. D.; Pumera, M. Chemically Reduced Graphene Contains Inherent Metallic Impurities Present in Parent Natural and Synthetic Graphite. Proc. Natl. Acad. Sci. U. S. A. 2012, 109 (32), 12899-12904.

(43) Wu, S.; Wen, G.; Liu, X.; Zhong, B.; Su, D. S. Model Molecules with Oxygenated Groups Catalyze the Reduction of Nitrobenzene: Insight into Carbocatalysis. Chem CatChem 2014, 6 (6), 1558-1561. (44) Zhang, J.; Wang, X.; Su, Q.; Zhi, L.; Thomas, A.; Feng, X.; Su, D. S.; Schlögl, R.; Müllen, K. Metal-Free Phenanthrenequinone Cyclotrimer as an Effective Heterogeneous Catalyst. J. Am. Chem. Soc. 2009, 131 (32), 11296-11297.

(45) Qi, W.; Liu, W.; Zhang, B.; Gu, X.; Guo, X.; Su, D. Oxidative Dehydrogenation on Nanocarbon: Identification and Quantification of Active Sites by Chemical Titration. Angew. Chem., Int. Ed. 2013, 52 (52), 14224-14228.

(46) Sánchez-Page, B.; Jiménez, M. V.; Pérez-Torrente, J. J.; Passarelli, V.; Blasco, J.; Subias, G.; Granda, M.; Alvarez, P. Hybrid Catalysts Comprised of Graphene Modified with Rhodium-Based NHeterocyclic Carbenes for Alkyne Hydrosilylation. ACS Appl. Nano Mater. 2020, 3 (2), 1640-1655.

(47) Espinosa, J. C.; Navalón, S.; Álvaro, M.; García, H. Reduced Graphene Oxide as a Metal-Free Catalyst for the Light-Assisted Fenton-Like Reaction. ChemCatChem 2016, 8 (16), 2642-2648.

(48) Wang, Y.; Xie, Y.; Sun, H.; Xiao, J.; Cao, H.; Wang, S. Efficient Catalytic Ozonation over Reduced Graphene Oxide for $p$ -Hydroxylbenzoic Acid (PHBA) Destruction: Active Site and Mechanism. ACS Appl. Mater. Interfaces 2016, 8 (15), 9710-9720.

(49) Wendlandt, A. E.; Stahl, S. S. Modular o -Quinone Catalyst System for Dehydrogenation of Tetrahydroquinolines under Ambient Conditions. J. Am. Chem. Soc. 2014, 136 (34), 11910-11913.

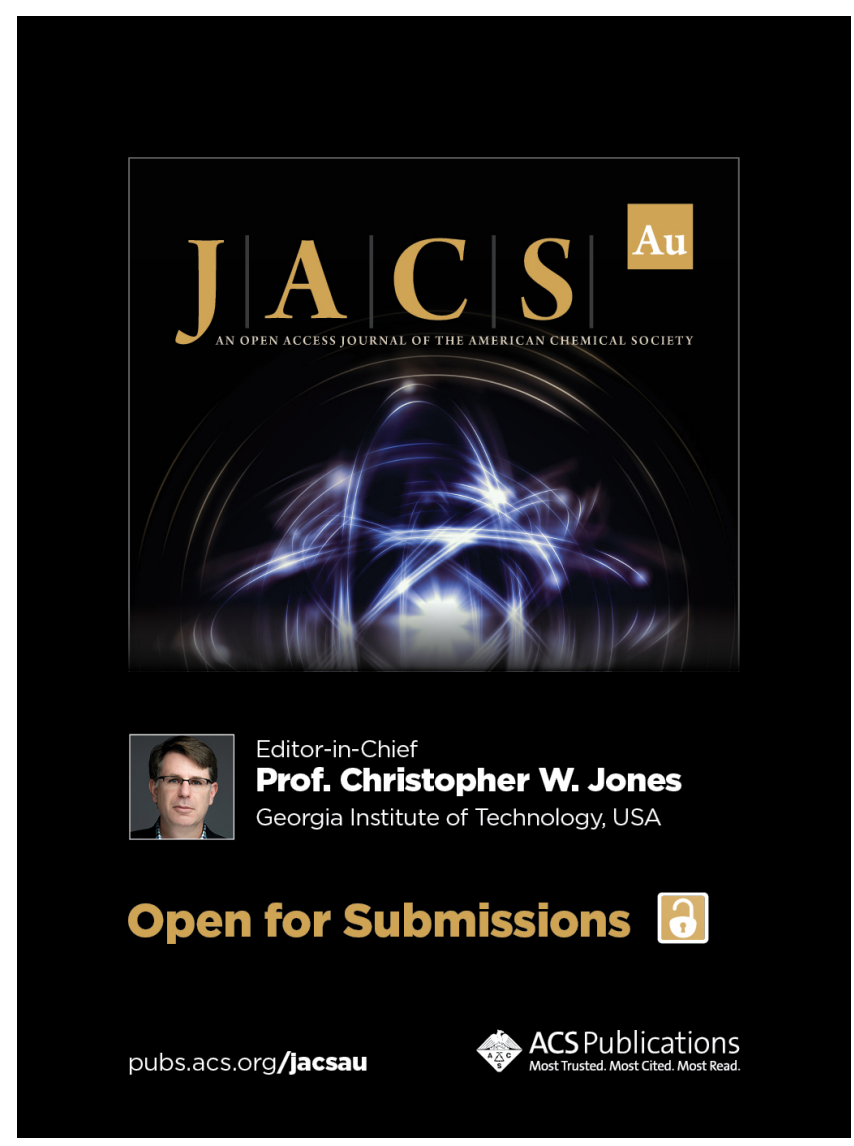

